\title{
El laberinto textual de la poesía de Luis Alberto de Cuenca: Los mundos y los días. Poesía 1970-2002
}

\author{
Luis Miguel SUÁREZ MARTíNEZ
}

IES Ornia

\begin{abstract}
RESUMEN
Este artículo analiza brevemente el proceso de reescritura de la obra poética de Luis Alberto de Cuenca, reflejado en los importantes cambios — desde la supresión de libros enteros hasta su fusión con otros - introducidos por el autor en las sucesivas ediciones de sus poesías completas. Este proceso de reescritura alcanza en Los mundos y los días. Poesía 1970-2002, la más reciente de sus recopilaciones, un grado mayor de complejidad, ya que, además de ofrecer nuevas variantes textuales, recupera ahora, en algunos libros, elementos de las ediciones originales suprimidos en las recopilaciones posteriores. De manera que, esta última edición de Los mundos y los días. Poesía 1970-2002 marca, también, un proceso de regresión textual.

Palabras clave: Luis Alberto de Cuenca, poesía, proceso de reescritura, Los mundos y los días. Poesía 1970-2002.
\end{abstract}

\begin{abstract}
This article examines the process de rewriting of Luis Alberto de Cuenca's poetry, reflected in the important changes - from the deletion of a whole book to its amalgamation with othersintroduced by the author in the successive collections of his complete poetry. This process of rewriting reaches in Los mundos y los días. Poesía 1970-2002, his most recent collection, a most degree of complexity, since, besides the new textual varianst, in somme books are got back elements of the original editions, deleted in later collections. So that the recent edition of Los mundos y los días marks a process of textual regression.
\end{abstract}

Keywords: Luis Alberto de Cuenca, poetry, process of rewriting, Los mundos y los días. Poesía 1970-2002.

Si se consulta el apartado bibliográfico que Juan José Lanz, en su edición crítica, dedica a consignar la obra poética publicada por Luis Alberto de Cuenca, se observará que los títulos allí enumerados sobrepasan la cincuentena ${ }^{l}$. Esto se debe a

${ }^{1}$ L. A. de Cuenca, Poesía. 1979-1996 (2006), pp. 145-147. 
que, aparte de los libros poéticos propiamente dichos - Los retratos (1971), Elsinore (1972), Scholia (1978), La caja de plata (1985), El otro sueño (1987), El hacha y la rosa (1993), Por fuertes y fronteras (1996; $2^{\mathrm{a}}$ edición aumentada 2002), Sin miedo ni esperanza (2002) y La vida en llamas (2006) - , se incluye en la lista bibliográfica todas las publicaciones poéticas del autor. Entre ellas, comenzando por las de formato breve, encontramos cuadernillos originales, que, salvo el caso de Necrofilia (1983), que mantendrá su carácter independiente, serán incluidos posteriormente en libros: por ejemplo, Seis poemas de amor (1986), que se integrará en El otro sueño, o Willendorf (1992) que hará lo propio en El hacha y la rosa. Otros cuadernillos tienen carácter antológico -generalmente temático- como Breviora (1984), Mitologías (2001), Ahora y siempre (2004)... Y aún existen otros más, fruto de encuentros poéticos, aulas de literatura, seminarios, etc. Y, como no podía ser menos, dada la afición entusiasta del autor por la materia, algunas ediciones para bibliófilos.

También forman parte de la lista bibliográfica ediciones de mayor formato y de naturaleza muy variada. Así, libros con poemas originales luego integrados en otros títulos: citemos, por ejemplo, El bosque y otros poemas (1997), anticipo, en su mayor parte, de Sin miedo ni esperanza (2002); o el más significativo Fiebre alta (1999), cuyas composiciones formarán parte de tres libros diferentes, Por fuertes y fronteras ( $2^{\mathrm{a}}$ edición aumentada), Sin miedo ni esperanza (2002) y La vida en llamas (2006) e incluso alguna composición allí incluida no forma parte aún de ninguno de los libros canónicos- El apartado bibliográfico se completa con antologías generales, como Doble filo (2001), y temáticas, como Vamos a ser felices y otros poemas de humor y deshumor (2003), De amor y amargura (2003) o Su nombre era el de todas las mujeres ( $1^{\circ}$ edición 2005$)$. A todo ello hay que añadir ediciones críticas - La caja de plata (2003), a cargo de J. Letrán y la del propio Lanz que tomábamos como punto de partida - , traducciones al italiano, ediciones mexicanas... y claro está las recopilaciones de su obra completa (tres hasta aquel momento).

Con posterioridad a la edición crítica de Lanz han aparecido - además de un nuevo libro canónico, El reino blanco (2010) - otros cuadernillos originales y antologías de muy variada índole ${ }^{2}$. Esta proliferación de ediciones pone de manifiesto el favor crítico y lector, y el prestigio del que goza hoy la poesía de Luis Alberto de Cuenca. Sin embargo, su abundancia aporta, por otra parte, una enorme complejidad textual, pues una de las características más acusadas del escritor madrileño es una constante labor de revisión de todos sus textos poéticos. Esta revisión es tan continua e intensa que, sobre todo en el caso de sus primeros libros, se debe hablar no de labor de corrección sino de reescritura.

Si tenemos en cuenta que algunos de sus poemas vieron la luz por primera vez en esos anticipos de libro, que muchos de los cuadernillos y de las antologías incluyen inéditos y que frecuentemente los poemas presentan variantes, a veces significativas, se comprenderá por qué señalábamos que esta proliferación de ediciones acarreaba una complejidad ecdótica considerable. Y si a todo ello añadimos que la obra poética

${ }^{2}$ Una bibliografía más actualizada de la poesía luisalbertiana puede verse ahora en L. A. de Cuenca, Filología y vida. Una antología (2010), pp. 241-243. 
de Luis Alberto de Cuenca abarca un lapso temporal de más de treinta años y que su estética actual está bastante alejada de la de sus primeros libros, publicados en la década de los setenta, el panorama al que debe enfrentarse quien pretenda llevar a cabo una edición crítica de esta poesía es arduo y trabajoso ${ }^{3}$; igualmente, puede resultar desconcertante para el lector encontrarse con poemas que cambian de libro, de título o de versos con frecuencia inusitada. En resumen, si toda edición crítica de un poeta aún vivo y en plena actividad creadora es siempre provisional, en el caso de De Cuenca lo es aún más, pues la labor limae, ha sido constante y el poeta se muestra en este aspecto sumamente exigente e infatigable.

Dadas estas circunstancias, las sucesivas recopilaciones de su obra poética constituyen un acontecimiento de la mayor importancia ${ }^{4}$, pues fijan en cada momento la situación textual del corpus poético, es decir, establecen lo que podemos denominar con toda propiedad su corpus canónico, que, como hemos advertido, está sometido a una continua variación. Los mundos y los días. Poesía 1970-2002, es la cuarta edición - la tercera bajo ese rótulo nervaliano - de la poesía completa del autor. De Cuenca había reunido su obra poética, por primera vez, en el volumen Poesía (1970-1989), publicado por la editorial sevillana Renacimiento en 1990, precedido de un breve prólogo del también poeta Julio Martínez Mesanza. La estructura externa del libro, que, además de los libros publicados hasta entonces, incluía dos secciones tituladas "Otros poemas", remarcaba ya la clara diferenciación que el autor establecía entre su obra lírica escrita hasta 1979, de estricta estética novísima (culturalismo exacerbado, refinamiento expresivo, irracionalismo...) — si bien Scholia es un libro de transición hacia la siguiente etapa - y la escrita con posterioridad a ese fecha (urbana, de lenguaje claro y coloquial, plena de humor y de ironía, etc.), que marcaba un giro significativo en su trayectoria para optar por una estética cercana a la poesía de la experiencia — por utilizar el término quizás más difundido- o de "línea clara", como la denomina el propio poeta; una línea poética, por cierto, que resultaba, en bastantes aspectos, antinovísima y a cuyo éxito contribuyó de forma decisiva el propio De Cuenca a partir de la publicación de La caja de plata.

Tras esta evolución parecía lógico, por tanto, que fueran los primeros libros —Los retratos (1971), Elsinore (1972), Scholia (1978)—, pertenecientes a una estética que parecía estar en los antípodas de la que para entonces, a finales de los

${ }^{3}$ Para comprobarlo basta con consultar las variantes textuales rigurosamente anotadas por J. J. Lanz en su edición. Y hay que tener en cuenta que la edición del profesor vasco abarca los cuatro libros centrales de la etapa de "línea clara" del autor, los cuales han experimentado una labor de reescritura bastante menos intensa que sus primeros poemarios, como podremos comprobar a lo largo de este trabajo.

${ }^{4}$ De hecho, la aparición posterior de esta nueva edición de Los mundos y los días ha provocado que la excelente edición crítica del profesor Lanz, fruto de un ímprobo y paciente trabajo de cotejo textual, no refleje ya - apenas un año después de su publicación - el último estadio de redacción de algunos libros y poemas luisalbertianos. Es el ejemplo más patente del carácter provisional al que está sometida toda edición crítica de la poesía luisalbertiana, dado su constante estado de variación. 
ochenta, defendía, los que sufran una transformación más importante. Los retratos desaparecía como libro, los cincuenta y dos poemas originales de Elsinore se reducían a veinticuatro y Scholia perdía cinco de los diecisiete poemas de los que constaba en la edición original. Los poemas conservados eran sometidos a diversas correcciones; entre ellas destacaba la supresión de la mayor parte de los elementos paratextuales (citas, epígrafes, notas explicativas...) que acompañaban a los poemas, elementos todos ellos que se venían a identificar con la estética novísima. Aunque parece evidente que las modificaciones textuales obedecían a criterios estéticos que ya no eran los mismos con los que se concibieron esos poemas en su origen, los poemas conservados de esta etapa novísima no eran siempre "los que reflejan una menor presencia de los afanes culturalistas de sus primeros libros"s. Más bien habría que inclinarse a pensar que el criterio seguido para seleccionar unos poemas y prescindir de otros era sobre todo su calidad estética - siempre a juicio del autor-, al margen de que las composiciones fueran más o menos culturalistas y estuvieran más o menos próximas al canon novísimo. La poesía conservada de esta primera etapa se completaba con un apartado, titulado "Otros poemas (1970-1979)", en el que se incluían un poema de Los retratos —el único vestigio conservado de aquel librocuatro poemas de Elsinore ${ }^{6}$ sometidos a tal transformación que solo en los títulos o en algunos versos recordaban los poemas originales y, por tanto, eran ya otros poemas distintos — de ahí que aparecieran en esta sección y no formando parte de Elsinore-; $\mathrm{y}$, finalmente, otros textos poéticos que no habían aparecido en ninguno de los libros poéticos publicados hasta aquellas fechas.

Se recogían a continuación las obras pertenecientes a la nueva "línea clara": el cuadernillo Necrofilia (1983), La caja de plata (1985) y El otro sueño (1987). El último apartado, "Otros poemas (1982-1989)" incorporaba textos aún no incluidos por entonces en un libro independiente y que, con posterioridad, pasaron a integrarse en El hacha y la rosa (1993). Las modificaciones textuales que afectan a estos libros son menos relevantes, aunque también existen correcciones diversas en los versos, algunos cambios de títulos o supresiones de poemas ${ }^{7}$. En conclusión, estas primeras

5 J. M. Barrajón, (1997), p. 115. Si hubiera sido así, poemas como "El crepúsculo sorprende a Roberto Alcázar en Charlotte Amalie", "Germania Victrix" o "South Wabash Avenue", por citar sólo tres ejemplos, no se hubieran conservado, pues a nuestro juicio, representan de forma paradigmática la estética novísima o veneciana del primer Luis Alberto de Cuenca. Para un análisis de esta primera edición de las poesías completas, vid. el mencionado trabajo de Barrajón.

${ }^{6}$ El poema conservado de Los retratos es "Alucinación de Santa Eulalia", pp. 71-72. En cuanto a los textos de Elsinore ahora reescritos son "Florencia", p. 73; "Angélica en la Isla del Llanto", p. 74; "Desitjada mort", p. 75 y "El muerto universal", p. 76. Este último es una nueva versión de "Amor y muerte en Calímaco de Cirene", que era el título con el que aparecía en Elsinore (1972), pp. 38-40. Todos ellos de una cierta extensión en la princeps de Elsinore se convierten ahora en breves poemas - podríamos hablar, con toda propiedad, de epigramas-, cuya extensión oscila entre los dos y los cinco versos.

${ }^{7}$ Para una síntesis de los cambios introducidos por De Cuenca en esta primera edición de su poesía completa, vid. Barrajón (1997), pp. 114-118. 
poesías completas ofrecían ya un primer ensayo del criterio corrector del poeta, fijaba, por primera vez, la obra que De Cuenca consideraba canónica — de ella desaparecía buena parte de su primera obra poética-y remarcaba, de forma significativa, el carácter de obra en marcha, es decir, en constante estado de revisión o reescritura.

En 1998, en la editorial Visor, veía la luz el volumen Los mundos y los días. Poesía 1972-1998, la segunda compilación — primera bajo este título- de su poesía completa. Al año siguiente aparecía una segunda edición que corregía algunas erratas de la primera y añadía un nuevo poema. Esta nueva recopilación — con dos ediciones - suponía, por un lado, una puesta al día bibliográfica, al añadir los libros publicados con posterioridad a Poesía 1970-1989: El hacha y la rosa (1993), Por fuertes y fronteras (1996) y El bosque y otros poemas (1997) —este último anticipo del posterior Sin miedo ni esperanza (2002), aunque tres de sus poemas se integrarían en la segunda edición de Por fuertes y fronteras (2002) - ; por otro lado, venía a fijar de nuevo el corpus canónico, que, como evidenciaban las cada vez más numerosas ediciones parciales de su obra (antologías, cuadernillos...), ya había variado respecto a Poesía 1970-1989.

Las variaciones introducidas en los primeros libros no eran muy numerosas pero sí resultaban muy significativas. Así, por ejemplo, Elsinore recuperaba otros dos poemas de la edición original y quedaba, ahora, compuesto por veintiséis, justo la mitad de su edición de 1972. Uno de ellos, "Angélica en la Isla del Llanto" (p. 16), que había sido reescrito en su práctica totalidad en Poesía 1979-1989 (p.74), volvía ahora prácticamente a su redacción primitiva de 1972. Scholia se incrementaba con un nuevo poema, "De y por Manuel Machado" (pp. 66-68), que no constaba en la edición original de 1978 y que había sido recogido en la sección "Otros poemas (1970-1979)" de Poesía 1970-1989 (pp. 82-84); esta sección desaparecía y, con ella, el único poema seleccionado de Los retratos (de ahí que la fecha que se toma en el título como punto de partida ya no sea 1970 sino 1972). Estos cambios conllevaban, a nuestro juicio, una redefinición del proceso de reescritura en dos aspectos: por un lado, las modificaciones introducidas en los sus primeros poemarios, a partir de ahora, no obedecían siempre a un simple proceso de selección sino también de adición, al añadir poemas no incluidos inicialmente en ellos — caso de Scholia - ; por otra, se cancelaban o rectificaban algunas de las modificaciones anteriores - caso ya señalado de "Angélica en la isla del llanto"-, con lo cual el proceso de reescritura no se mostraba siempre como una innovación textual sino que, a veces, consistía en una regresión, es decir, una vuelta a un estadio de redacción anterior.

Parte de esto ocurría también en los poemarios de "línea clara". Así, por ejemplo, en La caja de plata se mantenía la exclusión del poema "Europa" pero se recuperaba "Urganda la desconocida" (p. 119), trasladado anteriormente a El hacha y la rosa en su primera edición ${ }^{8}$. Los tres poemas de El otro sueño excluidos de Poesía 1970-1989 —“El perro Nicanor", "Julio Martínez Mesanza", "España"9 — volvían ahora a su

\footnotetext{
${ }^{8}$ El hacha y la rosa (1993), p. 17.

${ }^{9}$ El otro sueño (1987), pp. 130, 149 y 159.
} 
lugar original, al igual que "Sonja la Roja" (p. 135) que, con el título de "Red Sonja" se había reeditado en la edición original de El hacha y la rosa (p. 19). De este, a su vez, se excluía "Himno a la Virgen del Carmen". Con todo, en su conjunto, estos libros de la segunda etapa permanecían bastante próximos a sus respectivas primeras ediciones, por más que no faltasen cambios de menor calado: pequeños retoques en algunos versos o cambios de títulos ${ }^{10}$.

Esta tercera edición de Los mundos y los días, que apareció en 2007, ofrece, como cabía esperar, novedades importantes. Algunas de ellas ya las señala De Cuenca en la "Nota del autor" (pp. 9-10) que sirve de presentación al volumen: de esta recopilación de la obra completa se excluyen el primer libro Los retratos, como ha venido siendo habitual, y el más reciente, La vida en llamas (2006) (lo que significa, por otro lado, que propiamente no se trata de unas poesías completas); en Elsinore y Scholia, con profundas modificaciones, se rescatan poemas que no constaban en ediciones anteriores; La caja de plata y El otro sueño se agrupan ahora en un solo libro titulado La caja de plata; y aparecen por primera vez aquí la $2^{\mathrm{a}}$ edición ampliada de Por fuertes y fronteras (2002) y Sin miedo ni esperanza (2002), títulos ambos publicados con posterioridad a las dos primeras ediciones de Los mundos y los días (1998 y 1999). Veamos brevemente la naturaleza de tales modificaciones, comparándolas, sobre todo, con la ediciones de 1998 y 1999 de Los mundos y los dias.

Elsinore consta ahora de treinta y un poemas. El poema que lo inicia, "Alucinación de Santa Eulalia", formó parte originalmente de Los retratos ${ }^{11}$ y se convierte así en el único vestigio de aquel primer libro, cambiado ahora de ubicación. De la primitiva edición de 1972 se recuperan por primera vez para una recopilación y, por tanto, entran, por primera vez, en la obra canónica del autor "Virgo Potens" (p. 48), "El fin de Escaramouche", "The end", "Civitas dei" y "Ius primae noctis" (pp. 50-53). En cambio, se elimina el poema "Roland ofrece a Durendal y no a Aude como homenaje el último de sus pensamientos", que aparecía en las edición anterior de Los mundos y los días (pp. 19-20).

En cuanto a los retoques introducidos en los poemas conservados bajo el título de Elsinore, podemos encontrar cambios de títulos - "Pasión, muerte y resurrección de Propercio de Asís" se convierte en "Propercio y Cintia" (p. 26) - ; adiciones de versos — caso del verso 4 de "Marítima" (p. 22) - o supresiones - "Virgo Potens" (p. 48), por ejemplo, pierde el verso 9 que aparecía en la princeps ${ }^{12}$ - A veces, la supresión afecta a una sola palabra - la sustitución de "mas" por "pero" en el primer verso de la última estrofa de "South Wabash Avenue" (p. 43) -; pero el hecho puede ocasionar cambios métricos importantes: es lo que ocurre con "Jufré Rudel" (p. 24), en el que la supresión del artículo determinado que aparecía en ediciones anteriores al principio

${ }^{10}$ Para los cambios introducidos en los libros comprendidos entre Necrofilia (1983) y la $2^{\mathrm{a}}$ edición de Por fuertes y fronteras (2002), remitimos de nuevo a la edición crítica de Lanz (Vid. nota 1)

${ }^{11}$ Los retratos (1971), pp. 47-48.

${ }^{12}$ Elsinore (1972), p. 101. 
del último verso ("El llanto del agua") convierte el poema en un haiku métricamente riguroso (7-5-7). En otros casos, se trata de variaciones de sintagmas —así el v. 15 de "Germania Victrix" (p. 27) ${ }^{13}$ — o de versos casi enteros — por ejemplo, los versos 6-7 de "El crepúsculo sorprende a Roberto Alcázar en Charlotte Amalie" (p. 40) ${ }^{14}$. En fin, cambios como los ya reseñados pueden observarse en otros versos de "L.W.J." (p. 29), "Evocación de Francisco de Salas, cosmógrafo" (p. 39), etc ${ }^{15}$.

Aparte de estas modificaciones, existen otras tanto o más interesantes, a nuestro parecer, pues inciden en la recuperación de ciertos elementos propios de la estética novísima. Se trata de la restitución de algunas citas y epígrafes que acompañaban a los poemas en la edición original de 1972, y que hasta ahora no habían vuelto a aparecer en ninguna de las recopilaciones de la poesía completa del autor. Es el caso de "Here in the dark with you" (p. 21) o "Germania Victrix" (p. 27), que recuperan sus lemas latinos iniciales, o de "South Wabash Avenue" (p. 42), que recupera la advocación de Howard Hawks. Quizás, al restablecer estos elementos paratextuales, que quedaron en su momento como emblemas de la hojarasca culturalista, el autor considera ahora que no eran elementos tan meramente ornamentales y que pueden aportar alguna apoyatura al sentido del poema.

En Scholia, las novedades son igualmente interesantes. Se recuperan por primera vez dos textos de la edición original "Traum des Waldes" (pp. 59-60) y "Blanca ola" (pp. 67-68) ${ }^{16}$, ambos con numerosas correcciones —en especial, el segundo, ya que incluso se cambia el sentido de algunos versos de la cuarta estrofa: donde en la princeps se leía "Habrá un mañana. Y tú estarás en él. Ya estabas cuando echaron a suerte tus vestidos..." (p. 27) se lee aquí "Habrá un mañana. Sí. Tú no estarás en él. No estabas cuando..." (p. 68) - En cambio, se suprime el poema "6-IX-1956" que figuraba en todas las recopilaciones anteriores. Por último, se añaden dos poemas que no figuraban en la primera edición de Scholia: "Un milagro de Buda" (p. 78)-que había aparecido en Poesía 1970-1989 en la sección "Otros poemas (1970-1979)" (pp. 86-87) y que no presenta más novedades que la supresión de la dedicatoria a Eduardo Acosta y leves cambios de puntuación-y el soneto "Los arios a las puertas de la India” (p. 80), que no había sido incorporado a las poesías completas hasta ahora. Así

13 Desde la edición original de Elsinore se leía "En el trascoro tibio de un paisaje infernal"; ahora se lee "En el yermo infinito...".

14 "Murió feliz el ciervo acribillado por las ninfas, reflejando en sus ojos para siempre el desnudo impecable de Diana" sustituye a la lectura anterior "Murió feliz el ciervo con guirnaldas, con espejos / reflejando sus ojos para siempre en el escudo terso de la Aurora" (1999, p. 40).

${ }^{15}$ En "L.W. J.", por ejemplo, al comienzo del parágrafo 13, se lee "La muchacha se niega a entender a los pájaros", frente a la lectura "La muchacha rehúsa comprender a los pájaros" de todas las ediciones anteriores; en el penúltimo parágrafo de "Evocación de Francisco Salas, cosmógrafo" la lectura anterior "Era triste el lamento de tus pinceles en la bahía, / como una expedición a Maracaibo..." (1999, p. 39) se sustituye por "Era triste el lamento de tus pinceles en la bahía. / urge una expedición a Maracaibo...", etc.

${ }^{16}$ Vid. Scholia (1978), pp. 9-11 y 27-28, respectivamente. 
pues, la nueva versión de Scholia consta de dieciséis poemas, trece pertenecientes a la princeps y tres añadidos con posterioridad —además de los dos poemas ya señalados, "De y por Manuel Machado", insertado, como vimos, en Scholia desde la primera edición de Los mundos y los días-. En cuanto a los textos conservados de ediciones anteriores no contienen variantes muy significativas: leves correcciones presenta "La dama de Boston" (p. 63, vv. 1 y 3); levísimas correcciones, que afectan apenas a un sintagma, a alguna palabra o incluso a la puntuación, presentan "Rumbo a Londres, el conde Drácula resucita un pasado sentimental" (pp. 61-62), ) "Agag de Amaleq" (p. 72), The getaway" (p. 73) o "A. Persi Flacci choliambi” (p. 74).

Los poemarios de línea clara, como advierte el autor, presentan un estado de redacción bastante próximo a los originales: tres de los cuatro poemas del cuadernillo Necrofilia, han sido ligerísimamente retocados. Sin embargo, la novedad más importante la constituye la unión de La caja de plata y de El otro sueño en un solo libro que lleva el título del primero de ellos. Este cambio lo justifica el autor con una razón muy sólida, la unidad argumental y estética: "En realidad, — dice De Cuencael ciclo de La caja de plata se prolongó en El otro sueño, por lo que no puede hablarse de dos entregas líricas, sino de una sola" (p. 89) ${ }^{17}$. Por lo demás, La caja de plata presenta un estado de redacción prácticamente semejante al de su primera edición, pues el autor ha restituido incluso la nota y la dedicatoria que lo encabezaban en 1985 y ha recuperado el poema "Europa" (p. 141) que había sido eliminado de todas las recopilaciones de su poesía completa.

Lo mismo cabe decir de El otro sueño, que también recupera la nota y la dedicatoria que lo encabezaban en 1987. Los ligeros cambios introducidos, respecto a la redacción anterior, obedecen significativamente a un proceso de regresión textual. Es lo que ocurre con "Hoy he tenido un sueño con amigos", cuyos versos 39-40 presentaban redacciones distintas en todas y cada una de las compilaciones del autor, y que ahora recupera la versión de la $1^{a}$ edición de Los mundos y los días (1998), redacción que el poeta había modificado en la $2^{\mathrm{a}}$ edición que salió al año siguiente ${ }^{18}$. Yendo aún más allá en esta línea de regresión textual, "El perro Nicanor" (p. 162) se convierte ahora en poema heptasilábico, que era la estructura métrica original que ya tenía cuando se publicó por primera vez, en 1986, en la revista Zarza Rosa, antes de ser incluida en la $1^{\text {a }}$ edición de El otro sueño (1987). En esta primera edición, así como en las sucesivas poesías completas estaba compuesto en alejandrinos ${ }^{19}$.

${ }^{17} \mathrm{Y}$, en efecto, como ya han señalado críticos como J. Letrán (2007), pp. 19-20, ambos libros constituyen un ciclo poético que describe una trayectoria ascendente de superación de las tristezas anteriores para alcanzar lo que M. J. Conde Guerri (1988, p. 13) ha llamado una "estética matinal"; es decir, un tono vital optimista. En lo estético, por otra parte, este ciclo poético supone una depuración progresiva del lenguaje hacia un tono coloquial y una moderación notable de referencias culturalistas.

${ }^{18}$ Sobre los llamativos cambios introducidos sucesivamente por el autor en estos versos, vid. J. Letrán (2005), pp. 52-53, nota 58.

${ }^{19}$ Sobre este punto, vid. L. A. de Cuenca, Poesía 1979-1996 (2006), p. 245. 
Los tres últimos libros de la recopilación forman, en realidad, un segundo ciclo poético, dentro de la etapa de "línea clara". Frente al proceso de acercamiento a una estética matinal del ciclo anterior, este tríptico dibuja una trayectoria opuesta: el optimismo vital del sujeto poético se va tiñendo de melancolía en El hacha y la rosa, adquiere tonos más sombríos en Por fuertes y fronteras y, finalmente, desemboca en la profunda desolación de Sin miedo ni esperanza. En cierto modo, pues, este ciclo supone una vuelta al punto de partida, al inicio de La caja de plata ${ }^{20}$.

Las innovaciones textuales introducidas por el autor en este tríptico son, como ya hemos señalado, mínimas: en general, prescinde de las notas de autor que precedían a los libros y, salvo Por fuertes y fronteras, de las citas iniciales. Se añaden dedicatorias en algunos poemas o se suprimen en algún otro. Las variantes textuales de los poemas se limitan a pequeñas correcciones en algunos versos. Por ejemplo, cambio de orden de palabras: "corneja siniestra" sustituye la lección anterior "siniestra corneja" en el verso 9 de "El caballero, la muerte y el diablo" (p. 316). En otros casos, los leves cambios introducidos obedecen a una actualización contextual de las referencias del poema: así, en "La Sirenita" de Sin miedo ni esperanza, en sus versos 37-38 (p. 378), la referencia temporal a la historia de amor del sujeto poético - "que hoy sigue viva más de treinta meses / después", dice en la edición original de 2002 (p. 36) - se actualiza cronológicamente - "que hoy continúa viva tantos años / después"-; otros cambios obedecen a razones métricas: así en "El cuarto vacío" (p. 341) —uno de los textos añadidos en la segunda edición Por fuertes y fronteras ${ }^{21}$ - la sustitución de la palabra "habitación" por "alcoba" en el verso uno convierte la estructura métrica original del poema (7-7-5) en un haiku (5-7-5); o en "Visión de agosto" (pp. 381-382) de Sin miedo ni esperanza (pp. 39-40) se refunden los primitivos versos 24-25 en uno solo, con lo que se consigue una redistribución del número de versos que componen las cuatro estrofas del poema: así la distribución inicial 8-8-9-2 de la princeps se convierte en 8-8-8-2. Estos pequeños cambios ponen de manifiesto, una vez más, insistimos, el esmero y el extremo cuidado con que autor corrige sus versos, atento siempre al más mínimo detalle.

En resumen, esta nueva edición de Los mundos y los días insiste en ese proceso de reescritura al que el autor viene sometiendo constantemente su obra poética. El nuevo corpus canónico supone una recomposición de los libros que lo integran: Los retratos (1971) —en realidad, el único poema que de esta obra ha querido conservar el autor - se integra en Elsinore (1972); Scholia (1978), a la vez que recupera poemas de su edición original, incluye nuevos poemas que no constaban allí; La caja de plata (1985) se funde con El otro sueño (1987) y constituyen un único título. Y, en fin, en todos los libros se introducen correcciones de mayor o menor calado. Con todo, lo que más destacaríamos de esta tercera edición de Los mundos y los días es la

${ }^{20}$ Quizás sea esta perfecta trayectoria vital dibujada en los poemarios aquí recogidos una de las razones que ha llevado al poeta a cerrar esta recopilación con Sin miedo ni esperanza, renunciando a recoger el último poemario publicado hasta aquella fecha, La vida en llamas (2006).

${ }^{21}$ Por fuertes y fronteras (2002), p. 85. 
confirmación, en algunos casos, de un proceso de regresión textual: los libros de su primera etapa, respecto a las ediciones originales, recuperan e incluyen ahora un número de poemas mayor que en ninguna recopilación anterior; igualmente recuperan algunos elementos (citas, epígrafes...) eliminados en estadios intermedios, y se aproximan más a su edición original o princeps.

El resultado de este proceso de labor limae es un sugerente palimpsesto que pone de manifiesto la complejidad textual de la obra poética luisalbertiana. Cabría preguntarse qué hubiera ocurrido si este corpus poético, con tantas variantes de autor, algunas de ellas regresivas, perteneciera a tiempos pretéritos y hubiera estado sometido a los errores, deturpaciones y pérdidas propios de una transmisión manuscrita. La reflexión puede aportar interesantes consideraciones ecdóticas ${ }^{22}$.

\section{OBRAS CITADAS}

BARRAJÓN, Jesús María: "La poesía de Luis Alberto de Cuenca: diversa y semejante", en Revista de Literatura, 117, 1997, pp. 113-125.

CONDE GUERRI, María José: "Luis Alberto de Cuenca. El otro sueño", en Ínsula, 495, 1988, p. 13.

CUENCA, Luis Alberto de: Los retratos, Madrid, Azur, 1971.

CUENCA, Luis Alberto de: Elsinore, Madrid, Azur, 1972.

CUENCA, Luis Alberto de: Scholia, Barcelona, Antoni Bosch, 1978.

CUENCA, Luis Alberto de: Necrofilia, Madrid, Cuadernillos de Madrid, 1983.

CUENCA, Luis Alberto de: La caja de plata, Sevilla, Renacimiento, 1985.

CUENCA, Luis Alberto de: El otro sueño, Sevilla, Renacimiento, 1987.

CUENCA, Luis Alberto de: Poesía. 1970-1989, Sevilla, Renacimiento, 1990.

CUENCA, Luis Alberto de: El hacha y la rosa, Sevilla, Renacimiento, 1993.

CUENCA, Luis Alberto de: Por fuertes y fronteras, Madrid, Visor, 1996.

CUENCA, Luis Alberto de: Los mundos y los días. Poesía.1972-1998, Madrid, Visor, 1998 (2a edición, 1999).

CUENCA, Luis Alberto de: Por fuertes y fronteras ( $2^{\mathrm{a}}$ edición ampliada), San Sebastián de los Reyes, Universidad Popular José Hierro, 2002.

CUENCA, Luis Alberto de: Sin miedo ni esperanza, Madrid, Visor, 2002.

CUENCA, Luis Alberto de La vida en llamas, Madrid, Visor, 2006.

CUENCA, Luis Alberto de: Poesía. 1979-1996 (edición de Juan José Lanz), Madrid, Cátedra, 2006.

22 Por lo demás, si el panorama textual que hemos bosquejado resulta sumamente complejo, la reedición — con posterioridad a esta última entrega de Los mundos y los díasde Los retratos (2009) viene a complicarlo aún más. En efecto, este primer libro del poeta eliminado, según se ha visto a lo largo de este trabajo, en las todas las ediciones de su poesía completa - ha vuelto a ver la luz en edición exenta y en su redacción original. Esto supone modificar de nuevo la reestructuración de los libros canónicos que el poeta había realizado en esta $3^{\mathrm{a}}$ edición de Los mundos y los días. Así, pues, habrá que esperar con el máximo interés la siguiente edición de sus poesías completas para conocer su nuevo corpus canónico. 
CUENCA, Luis Alberto de: Los mundos y los días. Poesía. 1970-2002, Madrid, Visor, 2007.

CUENCA, Luis Alberto de: Los retratos, Madrid, Huerga y Fierro, 2009.

CUENCA, Luis Alberto de: Filología y vida. Una antología (edición de Luis Miguel Suárez), Ávila, Excelentísimo Ayuntamiento, 2010.

LETRÁN, Javier: La poesía postmoderna de Luis Alberto de Cuenca, Sevilla, Renacimiento, 2005.

LETRÁN, Javier: "La poesía de madurez de Luis Alberto de Cuenca", en Ínsula, 726, 2007, pp. 19-20. 